\title{
LETTER \\ Fast Coding-Mode Selection and CU-Depth Prediction Algorithm Based on Text-Block Recognition for Screen Content Coding
}

\author{
Mengmeng ZHANG ${ }^{\dagger a)}$, Member, Ang $\mathrm{ZHU}^{\dagger}$, and Zhi LIU ${ }^{\dagger}$, Nonmembers
}

\begin{abstract}
SUMMARY As an important extension of high-efficiency video coding (HEVC), screen content coding (SCC) includes various new coding modes, such as Intra Block Copy (IBC), Palette-based coding (Palette), and Adaptive Color Transform (ACT). These new tools have improved screen content encoding performance. This paper proposed a novel and fast algorithm by classifying Code Units (CUs) as text CUs or non-text CUs. For text CUs, the Intra mode was skipped in the compression process, whereas for non-text CUs, the IBC mode was skipped. The current CU depth range was then predicted according to its adjacent left $\mathrm{CU}$ depth level. Compared with the reference software HM16.7+SCM5.4, the proposed algorithm reduced encoding time by $23 \%$ on average and achieved an approximate $0.44 \%$ increase in Bjøntegaard delta bit rate and a negligible peak signal-to-noise ratio loss.

key words: screen content coding, text-block recognition, fast algorithm, coding-mode selection, depth prediction
\end{abstract}

\section{Introduction}

Screen content coding (SCC) is widely used in various applications, including wireless display, remote computer desktop access, and real-time screen sharing. SCC extensions that improve compression capabilities in videos containing a significant portion of rendered graphics, text, or animation rather than camera-captured video scenes have also been applied. SCC specifications were proposed in [1] during the 17th Joint Collaborative Team on Video Coding meeting in April 2014. These specifications are expected to be finalized in February 2016. In SCC, various compression modes/tools have been proposed to improve coding efficiency for screen content. These tools include the Intra Block Copy (IBC) mode, Palette-based coding (Palette) mode, and Adaptive Color Transform coding (ACT) mode [2]. When a Code Unit (CU) is coded in IBC mode, the prediction units (PU) of this CU find similar reconstructed blocks within the same picture. IBC can be considered as "motion compensation" within the current picture. The Skip and Merge modes in high-efficiency video coding (HEVC) inter coding are applied to the IBC mode. The Palette mode is an efficient method for representing blocks containing a small number of distinct color values. The mode enumerates such color values and sends an index for each sample to indicate to which color it belongs. The Palette mode can also be more efficient than the predictionthen-transform representation. In the ACT mode, a CU-level

Manuscript received January 28, 2016.

Manuscript publicized July 12, 2016.

†The authors are with North China University of Technology, Beijing, China.

a)E-mail: muchmeng@126.com

DOI: 10.1587/transinf.2016EDL8031 adaptation converts residuals to different color spaces to remove inter-color component redundancy [3], [4].

SCC presents a more outstanding performance and encoding time compared with HEVC for screen content because of these new tools. Many fast algorithms have been proposed to reduce computational complexity and conserve SCC encoding time. In [5], the authors used the edge direction based on the Sobel operator to decide on CU complexity. In [6], the authors calculated complexity based on CU variance. In [7], the authors proposed a fast-deciding CU partition mode algorithm based on entropy and coding bits for SCC. In [8], the researchers presented a fast intra coding approach for noiseless smooth regions in SCC based on the results of the statistical analysis of the boundary samples and the samples of noiseless smooth blocks. However, these algorithms only have a slight effect on text sequences.

Text content, as an important part of screen content, has significantly distinctive characteristics compared with other content. For text sequences, traditional Intra prediction achieves lower coding performance than IBC. However, the compression time of IBC is longer than that of Intra prediction. Therefore, if different coding modes could be skipped in some conditions, the encoding time would be reduced remarkably with minimal distortion. Under this argument, we classified the CUs as text or non-text CUs. In this regard, different kinds of CUs can skip the IBC or Intra mode compression processes based on histogram statistics in the proposed algorithm. We also simplified the compression procedure of $\mathrm{CU}$ by predicting the $\mathrm{CU}$ depth range.

We organized the remaining portion of this paper as follows. Section 2 presents our proposed algorithm. Section 3 discusses our experimental results. Finally, Sect. 4 concludes the paper.

\section{Proposed Fast Algorithm}

The proposed fast algorithm includes three parts: text-block recognition, coding-mode selection, and CU depth level prediction.

\subsection{Text-Block Recognition}

Text-block recognition considers a coding tree unit (CTU) size as a unit. All CUs in a text CTU would be marked as text CUs. In general, the characters of a line in a text CTU assume a lateral distribution. According to this characteristic, the part on text-block recognition includes three steps: 
a. Identify all the connected regions;

b. Find the bounding box of each region, and locate the center of the bottom edge of the bounding box in a binary image [9]. If the current block is a text block, the located pixels would display several parallel lines.

c. Use a Hough transform to determine the lines. For text sequences, almost all lines of text are horizontal. If the skew angle of these lines approximates 0 , the current block is considered as text CTU.

Hough transform is a method for identifying geometry in image processing. The simplest case of Hough transform is detecting straight lines. In Hough space, this paper uses the Hesse normal form (Eq. (1)) to perform this work ordinarily. According to the formula, $\omega$ is determined after all located pixels (located by step b) are processed.

$$
r=x \cos \omega+y \cos \omega
$$

where $r$ is the perpendicular distance from the origin to the line, and $\omega$ is the angle of that perpendicular line to the $x$ axis.

Some letters, such as $\mathrm{g}, \mathrm{j}$, and $\mathrm{y}$, would appear in a word. Therefore, $\omega$ has an error range of \pm 2 degrees for a text block as obtained from our experiments. Figure 1 displays the results of the text-block recognition. In this figure, a blue box represents a text CTU. Almost all the text blocks were recognized successfully as text CTUs. The background and camera-captured image blocks were recognized accurately as non-text CTUs. For some mixed blocks, the proposed algorithm for text-block recognition could sustain a negligible error, as determined by our experiments.

\subsection{Coding-Mode Selection}

The statistics of different modes with different $\mathrm{CU}$ depths are shown in Figs. 2 and 3 (data from the test sequence WebBrowsing_444) and used to analyze the attribute of the CU coding modes.

In Fig. 2, the CUs with IBC mode far outnumber those with the Intra mode for the text CUs. This result is achieved because the text content images are sharp and contain irregular edges. Consequently, the encoder cannot attain ideal coding efficiency when using only Intra prediction. However, this defect can be overcome by using IBC. The Intra

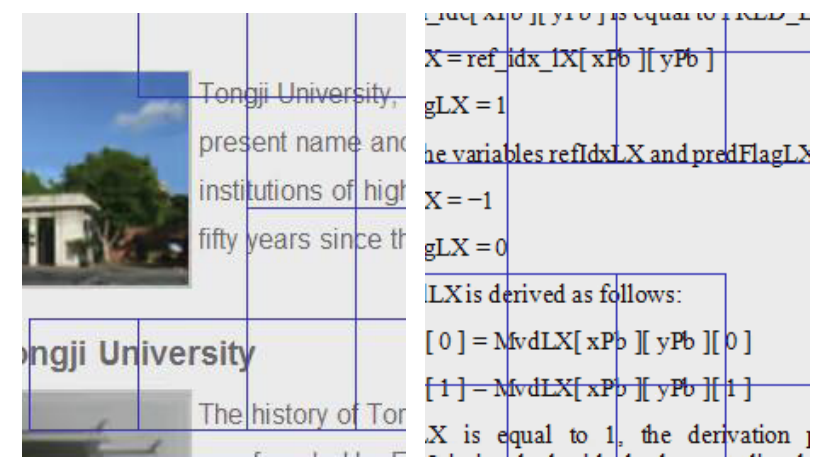

Fig. 1 Text-block recognition mode for text CUs can be skipped during the compression procedure under some conditions, which will result in shortened encoding time. By contrast, non-text CUs do not possess this character (Fig. 3). CUs with the Intra mode are more numerous than CUs with the IBC mode. A considerable portion of these CUs contains many camera-captured video contents in the sequence. For these camera-captured video CUs, the IBC mode has a small probability of being selected. Such CUs may also contain screen content blocks, such as background blocks. For these CUs, the IBC mode would also be selected. Hence, the IBC mode cannot be skipped absolutely for non-text CUs. The Luma histogram of the CUs of different modes were counted to identify further which mode could be skipped under certain conditions for different kinds of CUs. Figure 4 shows the histogram for the text CUs that utilized the Intra coding mode. Figures 5 and 6 display the histograms for the non-text CUs with IBC and Intra coding modes, respectively (data form WebBrowsing_444 and Desktop_444).

In Fig. 4, the majority of the Intra-mode CUs lie at depth level 3 on the left of the red line. At the left-hand side of the red line, the pixel value number (PVN) range of these CUs is 0 to 9. Thus, if the PVN of a text CU is greater than 9 , the CU could skip Intra prediction. Figures 5 and 6 are then compared as follows. If PVN is greater than 22 for a non-text CU, the IBC mode would not be selected. However, the Intra mode would still be chosen in the same case.

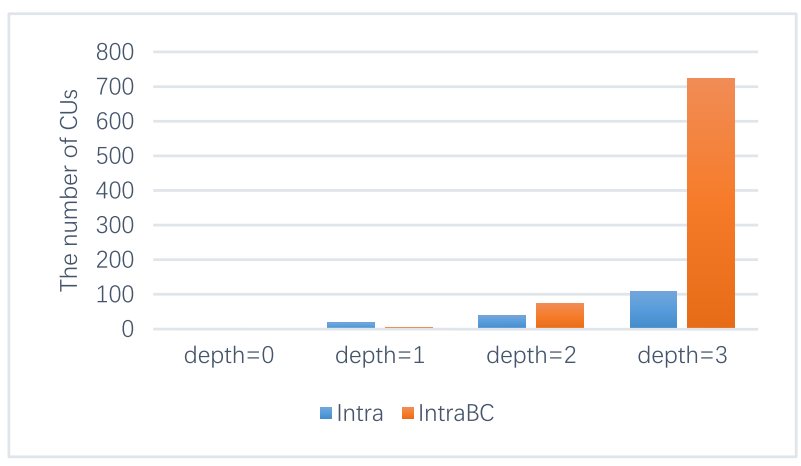

Fig. 2 Text CU statistics

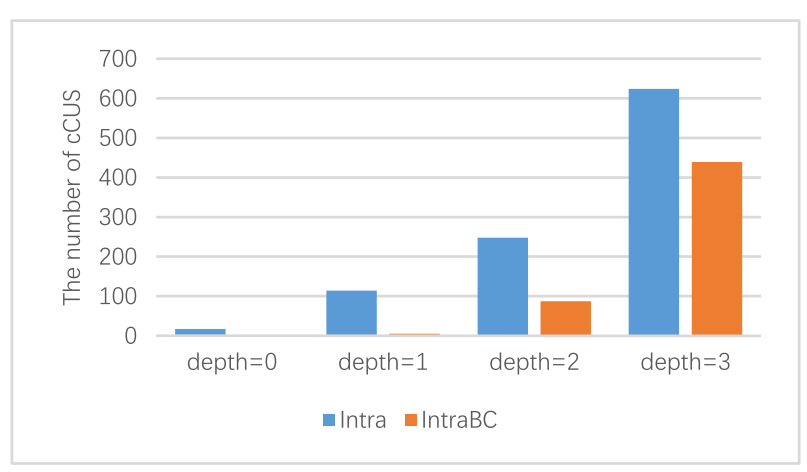

Fig. 3 Non-text CU statistics

In Figs. 2 and 3, e.g. Depth=1 denotes that the best depth level of this $\mathrm{CU}$ after the encoding procedure is 1 . 


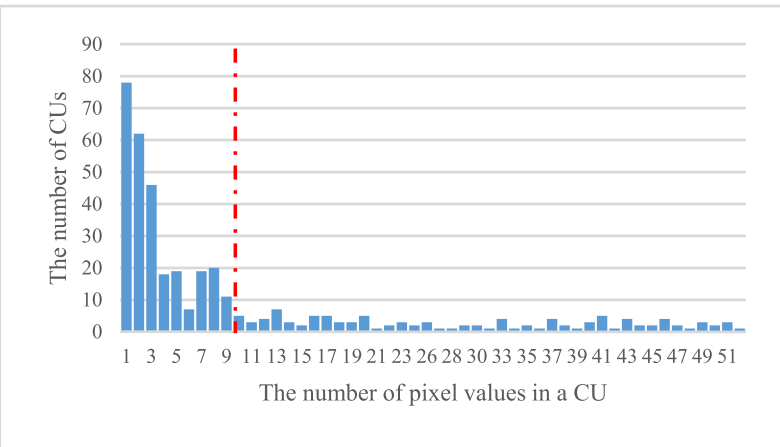

Fig. 4 Text CUs, with a depth level of 3, Intra mode

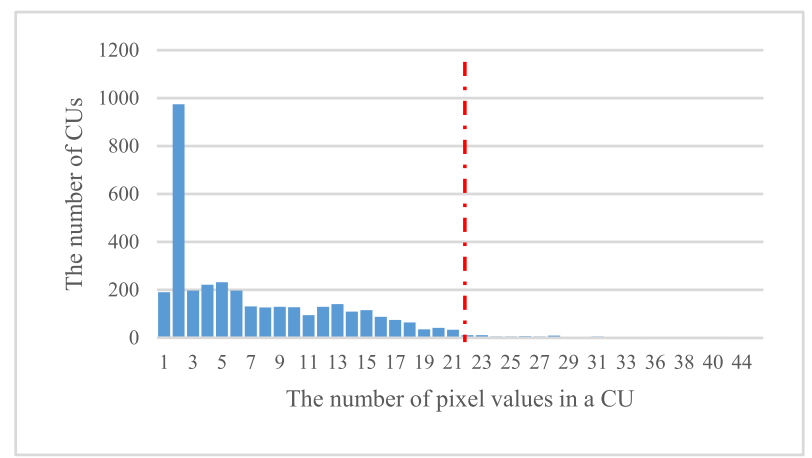

Fig. 5 Non-text CUs with a depth level of 3, IBC mode

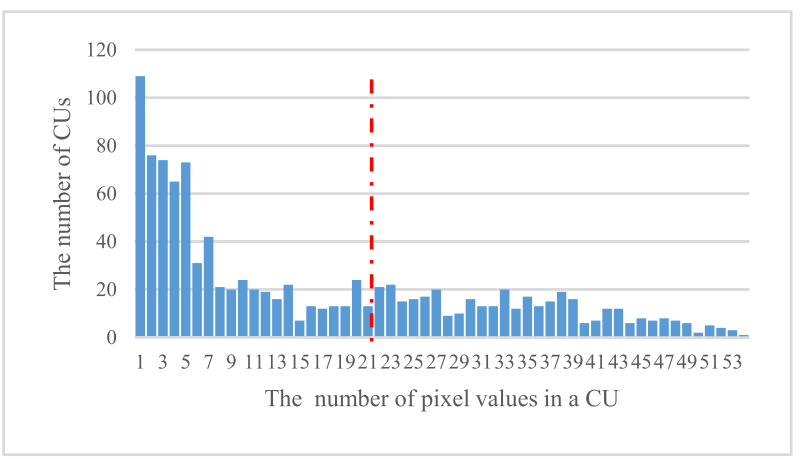

Fig. 6 Non-text CUs with a depth level of 3, Intra mode In Figs. 4-6, the horizontal axis represents the amount of pixel values in a $\mathrm{CU}$; the ordinate axis denotes the number of CUs that conforms to the characteristics.

Therefore, a threshold (T) can be set. If PVN is greater than T, IBC could be skipped for a non-text CU. However, CUs with the ACT, IBC Merge (except the case of CU with a depth level 0) and Palette modes do not have these characteristics. For this reason, the proposed algorithm could not be adapted to the latter mode selection.

Consequently, we obtained all thresholds with different depth levels. Table 1 shows the thresholds of the text CUs, whereas Table 2 displays those of the non-text CUs. Figure 7 presents the flowchart of the coding-mode selection.
Table 1 Text CU

\begin{tabular}{|l|l|l|}
\hline Depth Level & Coding-Mode Selection & Threshold(T) \\
\hline 0 & Skip IBC Merge & 3 \\
\hline 1 & Skip IBC & 15 \\
\hline 2 & Skip IBC & 27 \\
\hline 3 & Skip IBC & 29 \\
\hline
\end{tabular}

Table 2 Non-text CU

\begin{tabular}{|l|l|l|}
\hline Depth Level & Coding-Mode Selection & Threshold(T) \\
\hline 0 & Skip Intra & 2 \\
\hline 0 & Skip IBC Merge & 3 \\
\hline 1 & Skip Intra & 2 \\
\hline 2 & Skip Intra & 2 \\
\hline 3 & Skip Intra & 2 \\
\hline
\end{tabular}

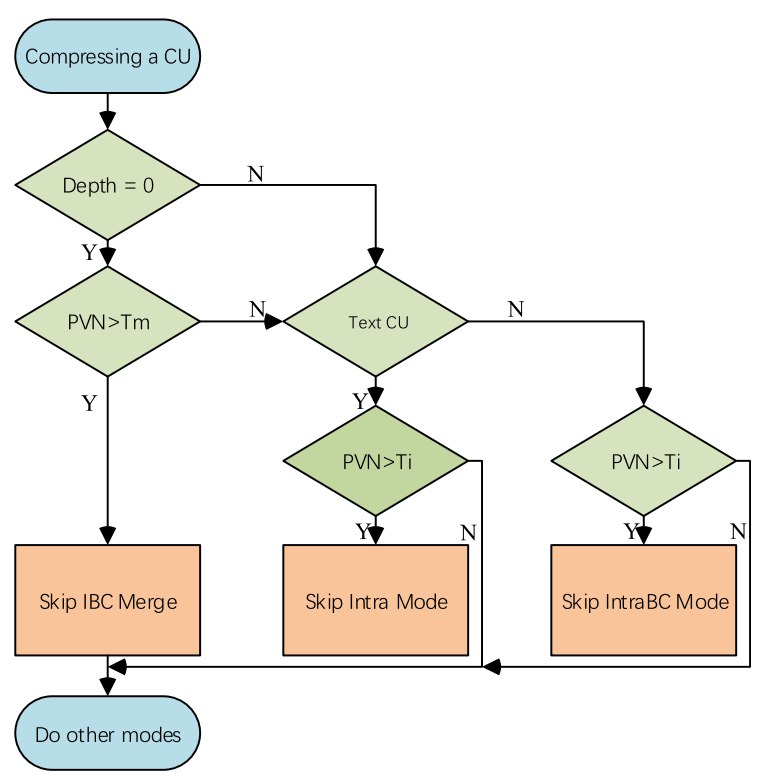

Fig. $7 T_{r u}=3, T_{i}$ represents a threshold in which the CU depth level is $i$.

\subsection{CU Depth-Level Prediction}

The prediction of the current CU depth level (CDepth) was based on the adjacent left CU depth level (LDepth).

First, a text CU and its adjacent left $\mathrm{CU}$ are both considered as text CUs. This paper analyzed all best depth levels of text CUs and its corresponding LDepth. Figure 8 shows the statistics of the test sequence WebBrowsing_444.

Figure 8 shows the following results. The ratio of $\mathrm{CDepth}=0$ is $0 \%$ when LDepth is equal to 1 . The ratio of CDepth $=0$ is $0 \%$ and CDepth $=2$ is $1 \%$ when LDepth is equal to 2. The ratio of CDepth $=0$ is $0 \%$ and CDepth $=1$ is $6 \%$ when LDepth is equal to 3 . Thus, when LDepth is 1 , the current CU with a depth level of 0 can skip check all coding modes. When LDepth has depths of 2 and 3, the current CU with depth levels of 0 and 1 could skip check all coding modes.

For non-text CUs, the algorithm mentioned above only applies to two neighboring CTUs with strong correlations. We assumed the following theory as true. If very high correlations between two adjacent CTUs exist, the $8 \times 8$ blocks 


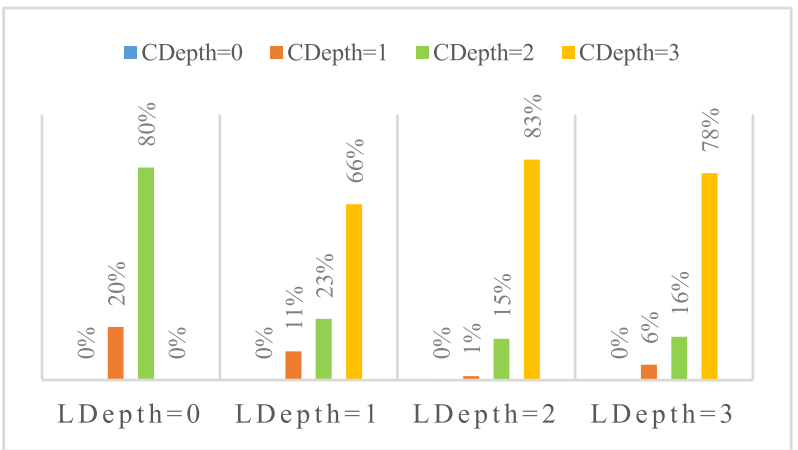

Fig. 8 CDepth and its corresponding LDepth for text CUs

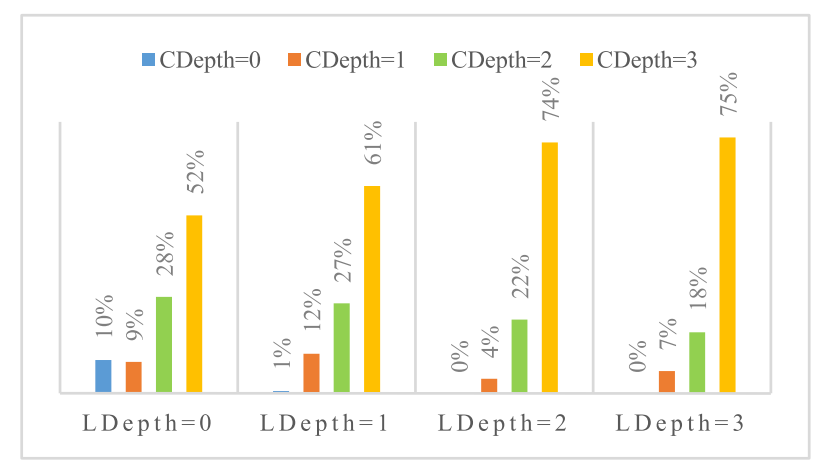

Fig.9 CDepth and its corresponding LDepth for text CUs

in the leftmost column of current CTU and in the rightmost column of the left CTU would have similar depth levels. In other words, the difference between the depth level of these adjacent blocks is equal to 0 , denoted as $D_{e}=0$.

Taking a CTU as a unit, we denoted the PVN of the current CTU as CP, the PVN of its adjacent left CTU as $\mathrm{LP}$, and the absolute difference value between CP and LP as $D P=a b s(C P-L P)$. This paper counts all DPs when the value of $D_{e}$ is 0 . For WebBrowsing_444, $D P=\{0,1,2,3$, $4,5,6,8,11,13,16,18,24,25,27,35,84,135\}$. The list of numbers 0 to 6 is continuous. These numbers are the best candidate values for DP because 7 is not included in this list. If we set the DP value to 8 , the prediction would incur a large error.

The different CDepths and their corresponding LDepths for the non-text CUs were counted to test the hypothesis. In this paper, the value of DP was set to 5. In the condition of $a b s(C P-L P) \leq 5$, a similar conclusion to that in Fig. 8 can be obtained (Fig. 9).

\section{Experimental Results}

We test our proposed algorithm on the HEVC test model HM-16.7+SCM5.4. The configuration is "All Intra Main." We evaluated the proposed algorithm with QPs 22, 27, 32, and 37 using five text sequences. One hundred frames of each sequence are coded to evaluate the performance of the proposed algorithm. Table 3 shows the experiment results.

The evaluation parameters are $\triangle T, \triangle P S N R_{-} Y$, and $B D$ -
Table 3 Tabulates the experimental result for all testing

\begin{tabular}{|l|l|l|l|}
\hline Sequences & $\Delta$ PSNR_Y & BD-rate_Y & $\Delta \mathrm{T}$ \\
\hline VideoConferencingDocSharing_444 & -0.03 & $0.28 \%$ & $22 \%$ \\
\hline ppt_doc_xls_444 & +0.05 & $0.28 \%$ & $20 \%$ \\
\hline Desktop_444 & -0.04 & $0.43 \%$ & $27 \%$ \\
\hline Console_444 & -0.10 & $0.32 \%$ & $21 \%$ \\
\hline WebBrowsing_444 & +0.03 & $0.91 \%$ & $29 \%$ \\
\hline Average & -0.02 & $0.44 \%$ & $23 \%$ \\
\hline
\end{tabular}

rate_Y (Eqs. (2) and (3)).

$$
\begin{aligned}
& \Delta T=\frac{T_{\text {anchor }}-T_{\text {proposed }}}{T_{\text {anchor }}} \times 100 \% \\
& \Delta P S N R_{Y}=P S N R_{\text {anchor }}-P S N R_{\text {proposed }}
\end{aligned}
$$

Sequences using Bjøntegaard delta PSNR, Bjøntegaard delta bitrate (BD-rate). The proposed algorithm enabled the reduction of encoding time by up to $23 \%$ and a BD-rate increase by $0.43 \%$.

\section{Conclusion}

This paper presented a novel and fast CU partition algorithm for alleviating the computational complexity of the SCM intra encoder for text sequences. In the proposed algorithm, text and non-text CUs were classified using textblock recognition. Some coding modes were skipped for different kinds of CUs during the compression processes. We also predicted the depth level of the current CU based on the depth level of its adjacent left CU. As demonstrated, the proposed algorithm is reliable and efficient in various text sequences.

\section{Acknowledgements}

This work is supported by the Natural National Science Foundation of China (No.61370111, No.61103113), Beijing Nova Programme (Z14111000180000), and Beijing Youth Talent Project (CIT\&TCD 201504001).

\section{References}

[1] R. Joshi and J. Xu, "HEVC Screen Content Coding Draft Text 1," Proc. 18th JCT-VC meeting, Sapporo, Japan, document JCTVCR1005, July 2014.

[2] H. Chen, A. Saxena, and F. Fernandes, "Nearest-neighbor Intra Prediction for Screen Content Video Coding," Proc. ICIP, pp.31513155, Oct. 2014

[3] J. Xu, R. Joshi, and R.A. Cohen, "Overview of the Emerging HEVC Screen Content Coding Extension,” Proc. IEEE Trans. Circuits Syst. Video Technol., p.1, Sept. 2015.

[4] Strasburg, FR, "CE2 Test 5: Unification of intra BC and inter modes using intra_bc_flag," Proc. JCTVC-S0112, Oct. 2014.

[5] M. Zhang and Y. Ou, "Edge Direction-based Fast Coding Unit Partition for HEVC Screen Content Coding," Proc. 21st International Conference, MMM 2015, pp.477-486, Jan. 5-7, 2015.

[6] J. Hou, D. Li, Z. Li, and X. Jiang, "Fast CU Size Decision Based on Texture Complexity for HEVC Intra Coding," Proc. MEC, pp.10961099, Dec. 2013.

[7] M. Zhang, Y. Guo, and H. Bai, "Fast intra partition algorithm for HEVC screen content coding," Proc. Visual Communications and 
Image Processing Conference, 2014 IEEE, pp.390-393, Dec. 2014.

[8] S.-H. Tsang, Y.-L. Chan, and W.-C. Siu, "Fast and efficient intra coding techniques for smooth regions in screen content coding based on boundary prediction samples," Proc. ICASSP, pp.1409-1413, April 2015.
[9] J.R. Parker, Algorithms for Image Processing and Computer Vision, Second ed., Wiley, 2011.

[10] H. Bai, C. Zhu, and Y. Zhao, "Optimized Multiple Description Lattice Vector Quantiza-tion for Wavelet Image Coding," Proc., vol.4, pp.912-917, CSVT, May 2007. 